\title{
Percepción del clima social en el aula por estudiantes de enfermería de tres facultades españolas
}

\section{Perception of the classroom social climate by nursing students in three Spanish Universities}

\section{Percepção do clima social na aula por estudantes de enfermagem de três faculdades espanholas}

\author{
R.M. Cárdaba-García ${ }^{a 1,2}$, M.S. Ovejero-de Pablo ${ }^{\mathrm{b} 1,3}$, \\ R. Soto-Cámara ${ }^{\mathrm{c} 1,4^{*}}$ \\ ORCID:

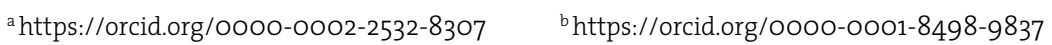 \\ ${ }^{c}$ https://orcid.org/0000-0002-9072-0364 \\ 'Salud Castilla y León, Gerencia de Emergencias Sanitarias, Valladolid, España \\ ${ }^{2}$ Universidad de Valladolid, Facultad de Enfermería-Campus Valladolid, Departamento de Enfermería, \\ Valladolid, España \\ 3Universidad de Valladolid, Facultad de Enfermería-Campus de Soria, Departamento de Enfermería, \\ Valladolid, España \\ ${ }^{4}$ Universidad de Burgos, Facultad de Ciencias de la Salud, Departamento de Ciencias de la Salud, Burgos, \\ España
}

Recibido: 27 abril 2019 / Aceptado: 30 noviembre 2019

RESUMEN

Introducción: El clima social en el ámbito académico es la percepción que tienen los individuos de los distintos aspectos del ambiente en el que se desarrollan. Contar con un clima social positivo facilita el rendimiento del alumnado y su satisfacción personal.

Objetivo: Analizar la relación del sexo, la edad o el centro universitario con la percepción del clima social en el aula en estudiantes del $2^{\circ}$ curso del Grado en Enfermería de las Facultades de Burgos, Soria y Valladolid, España.

Métodos: Estudio descriptivo correlacional, cuya población de estudio fueron todos los estudiantes matriculados en el $2^{\circ}$ curso del Grado en Enfermería en las Facultades de Burgos, Soria y Valladolid durante el periodo académico 2017/2018; se estudia la posible relación entre el clima social en el aula y los factores sociodemográficos mediante análisis bivariado. 
Resultados: Aceptaron participar 172 estudiantes. Se observó que tener más edad se relacionaba con una mayor motivación para asistir a clase $(p=0.009)$, con un sentimiento de orgullo ante la universidad ( $\mathrm{p}=0.001)$, con una mayor colaboración entre compañeros $(\mathrm{p}=\mathrm{0}$. o38) o con un mayor conocimiento de las normas de la universidad ( $\mathrm{p}=0.001$ ).

Conclusiones: El clima social en los centros analizados se sitúa en un nivel intermedio, es irrelevante el sexo y el centro universitario. El alumnado de mayor edad se presenta más motivado por el estudio, más colaborador y más identificado con el centro.

Palabras clave: Percepción; clima social; estudiantes de enfermería; normas; España.

\section{ABSTRACT}

Introduction: Academic social climate is the perception which individuals have regarding the diverse aspects of the environment in which they are academically developing. Having a positive social climate facilitates the performance and personal satisfaction among students.

Objective: To analyze the relationships between sex, age, and university with the perception of classroom social climate among students of the 2nd Grade of Nursing in the Universities of Burgos, Soria, and Valladolid, Spain.

Methods: This is a descriptive and correlational study. The population was constituted by all the students enrolled at the 2nd Grade of Nursing in the Universities of Burgos, Soria, and Valladolid during the 2017/2018 academic period. Using bi-variate analysis, the possible associations between classroom social climate and sociodemographic factors were studied. Results: A total of 172 students agreed to participate. It was observed that being older was associated to having a higher motivation to attend classes $(p=0.009)$, a higher feeling of pride towards the university $(p=0.001)$, a greater collaboration among colleagues $(p=0.038)$, or a better knowledge of the university norms ( $p=0.001)$.

Conclusions: Perceived social climate in these universities turn out to be fair. Sex and the specific university center were not particularly associated to the students' social climate perception. The older students tended to be more motivated and collaborative, and more identified with the university.

Keywords: Perception; social climate; nursing students; norms; Spain.

\section{RESUMO}

Introdução: O clima social no âmbito acadêmico é a percepção que têm os indivíduos dos distintos aspectos do ambiente no qual se desenvolvem. Contar com um clima social positivo facilita o rendimento do alunado e sua satisfação pessoal.

Objetivo: Analisar a relação do sexo, a idade ou o centro universitário com a percepção do clima social na aula em estudantes do $2^{\circ}$ curso de Grau em Enfermagem das Faculdades de Burgos, Soria e Valladolid, Espanha.

Métodos: Estudo descritivo correlacional, cuja população de estudo, foram todos os estudantes matriculados no $2^{\circ}$ curso de Grau em Enfermagem nas Faculdades de Burgos, Soria e Valladolid durante o período acadêmico 2017/2018; estuda-se a possível relação entre o clima social na aula e os fatores sociodemográficos mediante análise bivariada.

Resultados: Aceitaram participar 172 estudantes. Observou-se que ter mais idade relacionava-se com uma maior motivação para assistir à aula ( $\mathrm{p}=0.009)$, com um sentimento de orgulho ante a universidade ( $p=0.001)$, com uma maior colaboração entre companheiros ( $p=0.038$ ) ou com um maior conhecimento das normas da universidade $(p=0.001)$. 
Conclusões: O clima social nos centros analisados situa-se em um nível intermediário, é irrelevante o sexo e o centro universitário. O alunado de maior idade apresenta-se mais motivado pelo estudo, mais colaborador e mais identificado com o centro.

Palavras chave: Percepção; clima social; estudantes de enfermagem; normas; Espanha.

\section{INTRODUCCIÓN}

El clima social en el ámbito académico se refiere a la percepción que tienen los individuos de los distintos aspectos del ambiente en el que se desarrollan sus actividades educativas' ${ }^{1}$. Un clima social positivo sería aquel en el que el ambiente físico sea apropiado, se desarrollen actividades variadas y entretenidas, exista una comunicación respetuosa entre compañeros, entre alumnos y profesores, donde se fomente la escucha activa y la valoración de los participantes ${ }^{1,2}$.

Arón y Milicic ${ }^{3}$ han determinado que existen diferentes tipos de clima social en un grupo educativo, de forma que éste puede ser tóxico, nutritivo o invisible. Los climas tóxicos son aquellos en que el ambiente está contaminado con aspectos negativos, en donde aparecen el estrés y la ausencia de resolución de conflictos, lo que provoca un rendimiento académico deficiente. Los climas nutritivos, por el contrario, generan una convivencia social positiva en la que las personas se sienten cómodas, con disposición a aprender y cooperar. Los climas invisibles son aquellos que apenas tienen impacto en el grupo social; son poco frecuentes 4-9.

Garbanzo Vargas $^{10}$ en 2007 definió los factores asociados al rendimiento académico en estudiantes universitarios y concluyó que dicho rendimiento está condicionado por el contexto. Estos factores fueron clasificados en tres grupos: personales, sociales e institucionales. Dentro de los institucionales se reflejan dos de gran importancia relacionados con el clima social del grupo: a) el ambiente estudiantil, b) la relación entre estudiantes y profesores. El vínculo entre estudiantes, que se caracteriza por una marcada competitividad, suele ser negativa para el rendimiento académico. La solidaridad, el compañerismo y el apoyo social suelen describirse como elementos facilitadores. En la correlación entre estudiantes y profesor, los estudiantes desean encontrar en el profesor una relación tanto didáctica como afectiva, de manera que cuando sucede así, el rendimiento de este suele ser mejor ${ }^{2,10-12}$.

El rendimiento del alumnado ha sido objeto de estudio de la psicología positiva, cuyo modelo actual, ampliamente utilizado en las universidades españolas, se denomina PERMA, que es un acrónimo de las iniciales en inglés de sus cinco componentes: emociones positivas o vida placentera (positive emotions), compromiso (engagement), relaciones (relationships), significado o vida significativa (meaning) y logros (achievements) ${ }^{13-17}$.

El concepto de engagement se ha estudiado con mayor frecuencia en el ámbito académico, en donde se advierte una relación entre el rendimiento académico y el bienestar de la persona. Surgió de la mano de la Psicología Positiva de Seligman ${ }^{18}$, basada en el posicionamiento de los aspectos positivos laborales como eje central del trabajo. En los estudiantes universitarios es un predictor del buen desempeño de las tareas académicas y dicho engagement está influido por el ambiente que se tiene en el grupo social, en concreto, por medio del clima de trabajo, el apoyo social percibido y las oportunidades que se prestan para que el alumno aprenda. Además, los propios recursos personales del estudiante entran en juego en el proceso motivador por aprender ${ }^{19-21}$. Entre los recursos personales del alumno destaca la autoeficacia, la cual fue definida por Martínez y Salanova ${ }^{22}$ como la creencia de que se es capaz de algo en un ámbito concreto. Los estudiantes que se consideran poco eficaces en el afrontamiento de las demandas del entorno tienden a exagerar sus deficiencias y las 
dificultades que les ofrece el medio. Esta experiencia negativa de sí mismos les genera estrés y les distrae de los recursos disponibles a su alcance ${ }^{20,21}$.

A tenor de lo expuesto, parece claro que un buen clima en equipo y el reforzamiento de la autoeficacia pueden llevar al alumnado a un mejor rendimiento, mientras que, si existen altas demandas que produzcan estrés y se ofrecen pocos recursos, la motivación desciende notablemente y con ello aparece el agotamiento o burnout en los estudiantes. Cuando se produce burnout empeora el rendimiento, el alumno deja de comprometerse y evita involucrarse en el proceso de aprendizaje, por lo que evitarlo debería ser un objetivo prioritario en el profesorado ${ }^{2,22,23}$.

Cada vez es más frecuente el estudio del tipo de organización institucional en la que se encuentra inmersa el grupo educativo, ya que de la propia institución va a depender el tipo de clima que se genere en el aula. Bennett et al. ${ }^{24}$ desarrollan una teoría de organizaciones sanas, las cuales presentan las siguientes características: tienen en cuenta las dimensiones física, psíquica y social de los componentes del grupo; consideran el bienestar individual y grupal; se comprometen con éste a lo largo del tiempo; introducen en sus políticas el bienestar de los grupos y reconocen el esfuerzo que supone mantenerlo en los alumnos y profesores ${ }^{25}$.

De acuerdo con la literatura científica revisada, el clima social en las aulas del Grado de Enfermería es relevante no sólo en el ámbito académico, sino también para bienestar de la persona y, por tanto, influye en su calidad de vida; motivos más que suficientes para intentar develar cómo se relaciona el alumnado, si presentan un clima social que fomente el desarrollo de las personas y si consideran que la institución les es favorable. Describir este ámbito académico ha sido recomendado por otros investigadores previamente, tanto en Enfermería como en otros grados, para una vez conocida su relevancia, poder actuar en consecuencia ${ }^{25-27}$.

Consideramos, al igual que otros autores, que el segundo curso de un grado es el más adecuado para este estudio, porque ya se han desarrollado los lazos de amistad después de un curso académico y aún nos queda margen de acción en caso de tener que llevar a cabo medidas correctoras ${ }^{28-29}$.

Por lo anterior, se plantea como objetivo general analizar la relación entre el género, la edad o centro universitario y el clima social en el aula en estudiantes del $2^{\circ}$ curso del Grado en Enfermería de tres facultades.

Los objetivos específicos corresponden a describir el clima social en el aula y valorar la percepción que tienen los estudiantes respecto a éste.

\section{METODOLOGÍA}

Estudio descriptivo correlacional. La población diana fue de 275 estudiantes que estuvieran inscritos en alguna materia del $2^{\circ}$ curso del Grado de Enfermería, matriculados en el ciclo académico 2017/2018 en las Facultades de Enfermería Sede Burgos, Soria y Valladolid de la Universidad de Valladolid. Para la conformación de la muestra, se aplicó un cuestionario (62.5\% de respuesta), tras una clase teórica en horario lectivo, ésta quedó conformada por 172 estudiantes. Muestreo no aleatorio con participación voluntaria.

Para la obtención de datos se utilizaron los siguientes cuestionarios:

- Cuestionario autoadministrado exprofeso que recolecta los aspectos sociodemográficos principales del estudiante.

- Escala de Clima Social en el Aula para Universidades (ECSA_U), en su versión abreviada, validada para la población española por Rostán et $a .^{2}{ }^{28}$. Consta de 22 ítems, formulados en positivo, en los que el estudiante evalúa los tres factores más importantes del clima social 
(1. Relación entre los compañeros [9 ítems]; 2. Actuación del profesor y relación entre profesor-estudiantes [7 ítems]; 3 . Seguimiento de las normas y sentimiento de pertenencia [6 ítems]). La evaluación se realizó a través de una escala tipo Likert de 4 puntos, las opciones de respuesta fueron: nunca (0); a veces (1); bastantes veces (2); y siempre (3). La puntuación mínima que puede obtenerse es o, la máxima es 66 para la escala total; por factores el máximo es de 27, 21 y 18 puntos respectivamente. Las puntuaciones globales altas en la escala se relacionan con un mejor clima social en el grupo; a la inversa, las puntuaciones bajas indican peor clima social y los valores medios se relacionan con un clima social neutro. La fiabilidad de esta escala medida con alfa de Cronbach es de 0.84. Las variables consideradas fueron datos sociodemográficos (edad, sexo, sede de la universidad) y los resultados de la ECSA-U (puntuación total y por subescalas).

En el análisis de los datos se usó estadística descriptiva para caracterizar la muestra (frecuencia, distribución porcentual, medidas de tendencia central y dispersión). Para determinar la asociación entre las variables se utilizó la T de Student para muestras independientes y ANOVA de un factor o, bien, la correlación de Pearson, en función de la naturaleza de las variables. Se estableció un nivel de significación estadística de $\mathrm{p}<0.05$ para la interpretación de estos resultados. El procesamiento de datos se llevó acabo en el programa IBM SPSS v. 24.0 para Windows.

En todo momento, se tuvieron en cuenta los principios éticos, que garantizan la confidencialidad y anonimato de las respuestas de los participantes, ofreciéndoles la posibilidad de conocer los resultados una vez que el estudio se concluyera.

\section{RESULTADOS}

El total de alumnos que aceptaron participar en el estudio fue de 172. El 85.5\% corresponde a mujeres; la edad media de la muestra oscila entre los 20.9 años (DS 3.9), en un rango de 19 a 48 años. Por centros universitarios, $26.2 \%$ estudiaban en Burgos, $32.5 \%$ en Soria y $41.3 \%$ en Valladolid, con una tasa de participación entre el 75 y $53.8 \%$ por entidad.

En la ECSA-U, la puntuación total media (PTM) fue de 35.1 puntos (DS=9.4); en el factor 1. Relación entre los compañeros se obtuvo una media de 14.7 puntos ( $D S=4.4$ ); para el factor 2. Actuación del profesor y relación entre profesor-estudiantes los valores de la media fueron 11.4 puntos (DS=3.0); por último, la media en el factor 3 . Seguimiento de las normas y sentimiento de pertenencia correspondió a 8.9 puntos ( $\mathrm{DS}=3.2$ ).

Al analizar de forma individual los items de cada factor, se observó que los que obtuvieron una mayor puntuación media fueron dos ítems del factor 1, los cuales son interés de los estudiantes por formarse y ser profesionales competentes, así como respeto e interés de los estudiantes por sus compañeros del grupo; por el contrario, los que obtuvieron menor puntuación fueron facilitar la cooperación y ayuda entre los alumnos correspondiente al factor 2, y explicación al estudiante de lo que ocurre si incumple alguna norma del factor 3 (Tabla 1).

No se obtuvieron diferencias estadísticamente significativas al relacionar el sexo (Tabla 2), la edad (Gráfica 1) o el centro universitario (Tabla 3), con la puntuación total de la escala y con la de cada uno de los factores del ECSA-U. Se observó que los alumnos de mayor edad parecían estar más motivados por asistir a clase $(p=0.009)$, sentirse más orgullosos de la Universidad ( $p=0.001)$, colaborar entre compañeros con mayor frecuencia $(p=0.038)$ y tener un mayor conocimiento de las normas de la Universidad ( $\mathrm{p}=0.001)$. 
Tabla 1. Puntuación media y desviación estándar en cada uno de los ítems del cuestionario ECSA-U

\begin{tabular}{|c|c|c|}
\hline & PM & DS \\
\hline \multicolumn{3}{|l|}{ Relación entre los compañeros } \\
\hline Respeto e interés por los compañeros del grupo & 1.9 & 0.7 \\
\hline Buena relación entre los compañeros & 1.8 & 0.0 \\
\hline Interés a las aportaciones realizadas por los compañeros & 1.6 & 0.7 \\
\hline Acciones para evitar conductas de desprecio, discriminación o rechazo hacia otros compañeros & 1.7 & 0.9 \\
\hline Buena comunicación con los profesores & 1.5 & 0.6 \\
\hline Colaboración entre compañeros & 1.6 & 0.7 \\
\hline Debates entre estudiantes & 1.3 & 0.8 \\
\hline Interés por formarse y ser profesionales competentes & 2.0 & 0.7 \\
\hline Preocupación por el progreso del grupo & 1.4 & 0.7 \\
\hline \multicolumn{3}{|l|}{ Actuación del profesor y relación entre profesor-estudiante } \\
\hline Resolución de dudas de los estudiantes & 1.9 & 0.6 \\
\hline Respeto por creencias, ideas y sentimientos de otras personas & 1.8 & 0.7 \\
\hline Satisfacción por el logro de competencias de los alumnos & 1.7 & 0.8 \\
\hline Estudiantes motivados en asistir a clase & 1.5 & 0.7 \\
\hline Facilitar la cooperación y ayuda entre alumnos & 0.9 & 0.7 \\
\hline Acciones para facilitar el cumplimiento de las normas en el aula & 1.7 & 0.7 \\
\hline Preocupación de los profesores por los estudiantes y viceversa & 1.3 & 0.7 \\
\hline \multicolumn{3}{|l|}{ Seguimiento de las normas y sentimiento de pertenencia } \\
\hline Estudiantes se encuentran a gusto en la Facultad & 1.5 & 0.7 \\
\hline Estudiantes orgullosos de pertenecer a la Facultad & 1.5 & 0.8 \\
\hline Relación cordial entre estudiantes y profesores & 1.9 & 0.6 \\
\hline Facultad como espacio agradable & 1.8 & 0.7 \\
\hline Explicación al estudiante de las normas de funcionamiento de la Facultad & 1.3 & 1.0 \\
\hline Explicación al estudiante de lo que ocurre si un alumno incumple una norma en el aula & 1.1 & 1.0 \\
\hline
\end{tabular}

PM: Puntuación media - DS: Desviación estándar

Tabla 2. Puntuación media obtenida en la escala ECSA-U según sexo

\begin{tabular}{|c|c|c|c|}
\hline \multirow{2}{*}{ Factor } & \multicolumn{2}{|c|}{ Sexo } & \multirow{2}{*}{$\mathrm{p}$} \\
\hline & Varón & Mujer & \\
\hline & PM (DS)* & PM (DS) & \\
\hline Relación entre los compañeros & $13.9(4.7)$ & $14.9(4.4)$ & 0.3 \\
\hline Actuación del profesor y relación entre profesor-estudiante & $11.1(3.4)$ & $11.4(2.9)$ & 0.7 \\
\hline Seguimiento de las normas y sentimiento de pertenencia & $9.3(3.2)$ & $8.9(3.1)$ & 0.5 \\
\hline Puntuación TOTAL & $34.4(11.1)$ & $35.2(9.1)$ & 0.7 \\
\hline
\end{tabular}

* Puntuación media (Desviación estándar)

Tabla 3. Comparación de la puntuación media obtenida en la escala ECSA-U según centro universitario

\begin{tabular}{lcccc}
\hline \multirow{2}{*}{ Factor } & \multicolumn{3}{c}{ Centro universitario* } & p \\
\cline { 2 - 4 } & UBU & USO & UVA & \\
\hline Relación entre los compañeros & $15.6(4.5)$ & $14.5(4.2)$ & $14.4(4.6)$ & 0.3 \\
\hline Actuación del profesor y relación entre profesor-estudiantes & $11.7(3.3)$ & $10.9(2.4)$ & $11.5(3.2)$ & 0.4 \\
\hline Seguimiento de las normas y sentimiento de pertenencia & $9.5(3.2)$ & $9.0(3.3)$ & $8.5(3.4)$ & 0.3 \\
\hline Puntuación TOTAL & $36.8(10.1)$ & $34.4(8.5)$ & $34.4(9.7)$ & 0.3 \\
\hline
\end{tabular}

* UBU: Universidad de Burgos - USO: Universidad de Soria - UVA: Universidad de Valladolid 


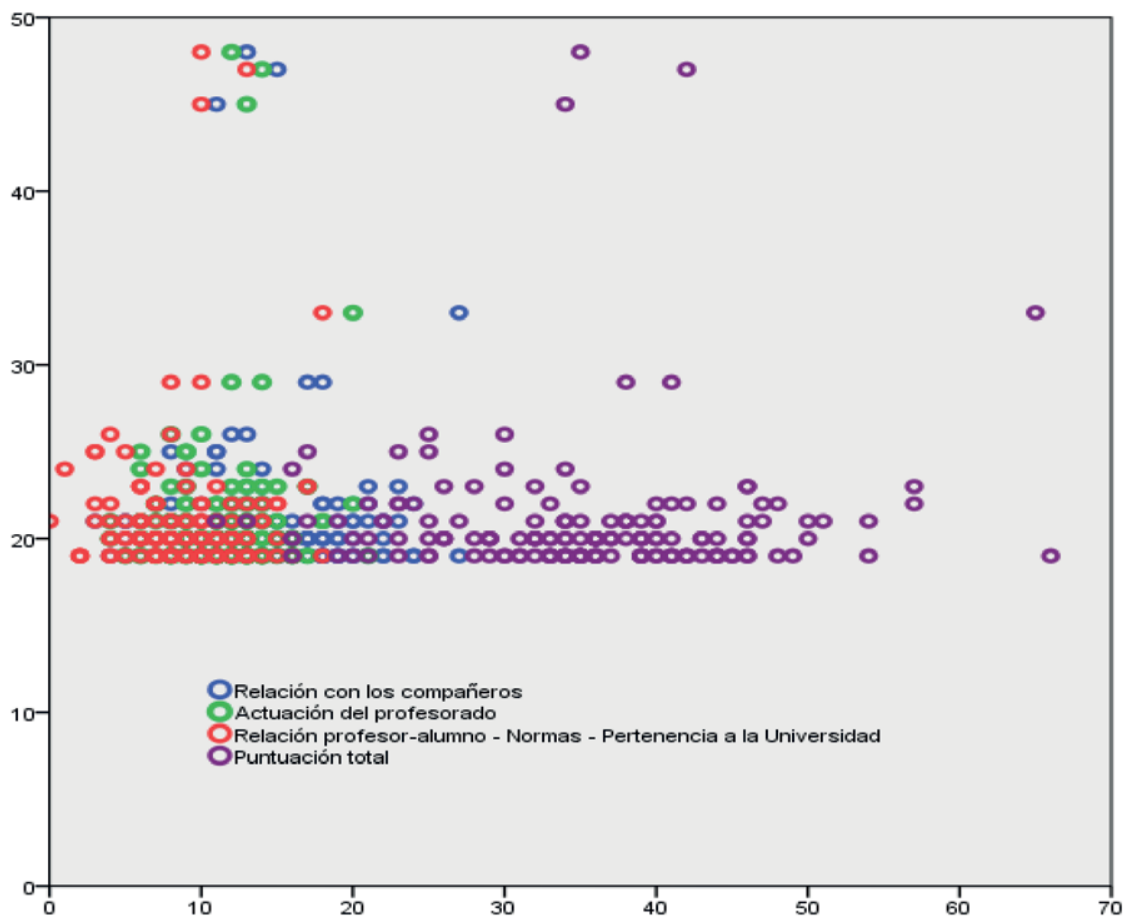

Gráfica 1. Comparación de la puntuación media de la escala ECSA-U obtenida según edad

\section{DISCUSIÓN Y CONCLUSIONES}

El clima social es un aspecto clave de la calidad docente en las universidades, de acuerdo con Moreno et al. ${ }^{29}$. En esta investigación se obtuvieron valores medios tanto en la puntuación global como en los factores del clima social, lo que permite afirmar que, en las Facultades estudiadas el clima es intermedio. Al tener en cuenta la puntuación media global en la evaluación de clima social y, de acuerdo con otros estudios, se plantea la posibilidad de mejorar dicho clima, para conseguir una adecuada convivencia en el aula y aumentar el rendimiento del alumnado o engagement ${ }^{17,19,30}$; es importante entender esta mejora como un objetivo que debe ser desarrollado en todos los cursos académicos de las Facultades que forman parte de la investigación y que podría extenderse a otras.

En esta investigación, con la variable sexo no se observó relevancia en cuanto a la percepción que el alumnado tiene sobre las relaciones con los compañeros, lo cual no ha ocurrido en otras investigaciones, en las que ser mujer o ser hombre influye notablemente en ello ${ }^{25,26}$. La discrepancia entre nuestro resultado y el de otras investigaciones motivan a los autores a realizar proyectos orientados a comprobar este hallazgo.

Aspectos del clima social en el aula como la colaboración o la ayuda mutua fueron mejor valorados por los estudiantes de mayor edad; la literatura científica consultada afirma tal punto, pero no se había encontrado hasta este momento la emoción de orgullo de pertenencia a la Universidad en relación con la edad del estudiante ${ }^{21}$. El grupo de investigación plantea, de acuerdo con el resultado mostrado, la posibilidad de elaborar un programa de orientación a nuevos alumnos, basado en sesiones breves que sean llevadas a cabo por los estudiantes de más edad en cursos más avanzados, para que de ese modo se transmita su entusiasmo por el centro.

El centro universitario no influye en la percepción de los alumnos en cuanto al clima en el aula. En los estudios revisados no se ha encontrado ninguno multicéntrico como éste, de modo que no 
se puede comparar dicho resultado con otros estudios del mismo tipo, pero si se puede cotejar que, en general, los resultados en la escala ECSA-U en otros centros son similares a los encontrados en el presente trabajo. Cierto es que los contextos de otras investigaciones son similares al actual, por lo que se requieren estudios en contextos diferentes que establezcan exactamente su implicación en la percepción del clima social ${ }^{23}$.

La propuesta más común en caso de un clima no óptimo, como los resultados obtenidos en esta investigación, es proponer estrategias tendientes a alcanzar un clima social nutritivo, que es el que permite el mayor desarrollo de competencias en el alumnado y una mayor satisfacción laboral del personal docente; de manera que los centros universitarios que se han evaluado, son candidatos a la aplicación de un programa de mejora del contexto, por ejemplo, el de Aulas Felices que fue elaborado por el Equipo SATI de Zaragoza en 2010, apoyándose en la perspectiva de la educación positiva ${ }^{4,10,31}$.

El hecho de que el estudiante valore de manera positiva el interés por formarse y poder ser un profesional competente en el futuro, hace patente la individualidad de su proyecto profesional; lo que no está reñido con el respeto que tiene hacia sus compañeros y su interés por no dañar al resto del alumnado. El puntaje bajo en ayuda y cooperación con el resto de alumnos es una muestra más de esa individualidad, de forma que no se pretende molestar al otro, pero tampoco se quiere ofrecer ayuda, por lo que la actitud predominante es de neutralidad. Esto nos lleva a pensar en la necesidad de fomentar de manera transversal en todas las asignaturas del Grado de Enfermería el trabajo en equipo, tanto a nivel teórico, por medio del fomento del Aprendizaje Basado en Problemas (ABP), como práctico, a través de grupos reducidos de trabajo en actividades de aula. Es destacable la baja puntuación obtenida en el ítem explicación al estudiante de lo que ocurre si se incumple una nor$m a$, ya que esta reflejaría falta de orientación al alumno. Se necesitarían más estudios para obtener una explicación razonada a este hecho, pero puede deberse a la ausencia de consecuencias en las Facultades para sancionar el incumplimiento de normas ${ }^{26}$. De ser así, sería adecuado que todo el alumnado del Grado, desde el primer curso, académico tuviera acceso a las normas institucionales y a las responsabilidades o sanciones que pudieran recibir por el incumplimiento de éstas.

Entre las principales limitaciones del estudio, se encuentra el hecho de que se analiza un reducido número de variables sociodemográficas, o el haberse limitado a un único curso académico.

\section{RESPONSABILIDADES ÉTICAS}

Protección de personas y animales. Los autores declaran que para esta investigación no se han realizado experimentos en seres humanos ni en animales.

Confidencialidad de los datos. En este artículo no aparecen datos confidenciales de los participantes. Derecho a la privacidad y consentimiento informado. Todos los participantes otorgaron previamente su consentimiento, se respetó en todo momento la confidencialidad de los datos obtenidos, los cuestionarios fueron anónimos.

Financiamiento. No se recibió ningún tipo de financiamiento para la presente investigación.

Conflicto de intereses. Los autores declaran la ausencia de posibles conflictos de intereses con respecto a la investigación, autoría y/o publicación de este documento.

Agradecimientos. Los autores agradecen la colaboración voluntaria y desinteresada del alumnado de segundo curso del Grado de Enfermería de las Universidades de Burgos, Soria y Valladolid, sin cuya participación esta investigación no hubiera sido posible. 


\section{REFERENCIAS}

1. Arón-Svigilsky AM, Neva-Milicic M. Clima social escolar y desarrollo personal: un programa de mejoramiento. Santiago de Chile: Editorial Andrés Bello; 1999.

2. Arón-Svigilsky AM, Neva-Milicic M, Armijo I. Clima Social Escolar: una escala de evaluación-Escala de Clima Social Escolar, ECLIS-. Univ. Psychol. 2012; 11(3): 803-13. http://bit.ly/34318dm

3. Aron AM, Milicic N. Climas Sociales Tóxicos y Climas Sociales Nutritivos para el Desarrollo Personal en el Contexto Escolar. Psykhe. 2000; 9(2): 117-23. https://doi.org/10.11144/Javeriana.upsy11-3.csee

4. Zubieta EM, Delfino GI. Satisfacción con la vida, bienestar psicológico y bienestar social en estudiantes universitarios de Buenos Aires. Anu. Investig. - Fac. Psicol., Univ. B. Aires. 2010; 17: 277-83. http://bit.ly/2us5v3N

5. Berger-Silva C, De Macedo-Lisboa C. Violencia escolar: estudios y posibilidades de intervención en Latinoamérica. Santiago de Chile: Editorial Universitaria; 2009.

6. Milicic N, López de Lérida S. Hostigamiento escolar: propuestas para la elaboración de políticas públicas. Santiago de Chile: Vicerrectoría de Comunicaciones y Asuntos Públicos, Pontificia Universidad Católica de Chile; 2008.

7. Goleman D, González-Raga D. Inteligencia social: la nueva ciencia de las relaciones humanas. Barcelona: Kairos; 2006.

8. Castro-Pérez M, Morales-Ramírez ME. Los ambientes de aula que promueven el aprendizaje, desde la perspectiva de los niños y niñas escolares. Educare. 2015; 19(3): 1-32.

http://dx.doi.org/10.15359/ree.19-3.11

9. McEvoy A, Welker R. Antisocial behavior, academic failure, and school climate: A critical review. J Emot Behav Disord. 2000; 8(3): 130-40. https://doi.org/10.1177\%2F106342660000800301

10. Garbanzo-Vargas GM. Factores asociados al rendimiento académico en estudiantes universitarios, una reflexión desde la calidad de la educación superior pública. Educación. 2007; 31(1): 43-63.

https://doi.org/10.15517/revedu.v3111.1252

11. Ruvalcaba-Romero NA, Gallegos-Guajardo J, Fuerte-Nava JM. Competencias socioemocionales como predictoras de conductas prosociales y clima escolar positivo en adolescentes. Rev. interuniv. form. profr. 2017; 31(1): 77-90. http://bit.ly/37wZeBb

12. Gallardo G, Reyes P. Relación profesor-alumno en la universidad: arista fundamental para el aprendizaje. Calid. educ. 2010; (32): 78-108. http://dx.doi.org/10.31619/caledu.n32.152

13. Umucu E, Wu JR, Sanchez J, Brooks JM, Chiu CY, Tu WM, et al. Psychometric validation of the PERMAprofiler as a well-being measure for student veterans. J Am Coll Health. 2019.

https://doi.org/10.1080/07448481.2018.1546182

14. Domínguez-Bolaños RE, Ibarra-Cruz E. La psicología positiva: Un nuevo enfoque para el estudio de la felicidad. Razón y Palabra. 2017; 21(1): 660-79. http://bit.ly/2tUV2gW

15. Barahona-Esteban MN, Sánchez-Cabaco A, Urchaga-Litago JD. La Psicología Positiva aplicada a la educación: el programa CIP para la mejora de las competencias vitales en la Educación Superior. REFIEDU. 2013. 6(4): 244-56. http://bit.ly/2rXOU6g

16. Seligman MEP, Csikszentmihalyi M. Positive psychology: An introduction. Am Psychol. 2000; 55(1): 5-14. https://doi.org/10.1037/0003-066X.55.1.5

17. Fernández-Ríos L, Vilariño-Vázquez M. Historia, investigación y discurso de la Psicología Positiva: Un abordaje crítico. Ter. psicol. 2018; 36(2):123-33.https://doi.org/10.4067/So718-48082018000200123

18. Seligman MEP. Learned optimism: How to change your mind and your life. New York: Pocket Books; 1998. 
19. Prieto-Egido M. La psicologización de la educación: Implicaciones pedagógicas de la inteligencia emocional y la psicología positiva. Educación XX1. 2018; 21(1): 303-20.

https://doi.org/10.5944/educxx1.20200

20. Haro-Soler MM. ¿Cómo desarrollar la autoeficacia del estudiantado? Presentación y evaluación de una experiencia formativa en el aula de traducción. Rev. Digit. Invest. Docencia Univ. 2017;11(2): 50-74. http://dx.doi.org/10.19083/ridu.11.567

21. Bolaños-Medina A. Self-efficacy in translation. Translation and Interpreting Studies. 2014; 9(2):197218. https://doi.org/10.1075/tis.9.2.03bol

22. Martínez-Martínez IM, Salanova-Soria M. Autoeficacia en el trabajo: El poder de creer que tu puedes. Estudios Financieros. 2006; 45(279): 175-202. https://bit.ly/2HAWA2V

23. Montero-Rojas E, Villalobos-Palma J, Valverde-Bermúdez A. Factores institucionales, pedagógicos, psicosociales y sociodemográficos asociados al rendimiento académico en la Universidad de Costa Rica: Un análisis multinivel. RELIEVE. Rev. electrón. investig. eval. educ. 2007; 13(2): 215-34. http://bit.ly/2wpb57L

24. Bennett JB, Cook RF, Pelletier KR. Toward an integrated framework for comprehensive organizational wellness: Concepts, practices and research in workplace health promotion. En: Quick JC, Tetrick LE (Eds). Handbook of occupational health psychology. Washington DC, US: American Psychological Association; 2003. p. 69-95. http://dx.doi.org/10.1037/10474-004

25. Pacheco-Castilla J. Estrés académico de los estudiantes de enfermería en una Universidad privada de Puerto Rico y su asociación con el rendimiento académico. [Tesis]. Málaga, España: Facultad de Ciencias de la Salud, Universidad de Málaga; 2017. https://bit.ly/32cSiHK

26. Suárez-Castellón K. Clima social y vivencias académicas en estudiantes de una universidad de Cartagena. [Tesis]. Cartagena de Indias, Colombia: Facultad de Educación, Ciencias Humanas y Sociales, Universidad de San Buenaventura; 2015. http://bit.ly/2OVPxbd

27. Araújo-Rodrigues B, Almeida LS, Paúl MC. Transição e Adaptação académica dos estudantes à escola de enfermagem. Revista Portuguesa de Psicossomática. 2003; 5(1): 56-64. http://bit.ly/2SxWPSK

28. Rostán-Sánchez C, Cañabate-Ortiz D, González-Carrasco M, Albertín-Carbo P, Pérez-Burriel M. Una herramienta para evaluar el clima social del aula en entornos universitarios. Rev. electrón. investig. psicoeduc. psicopedag. 2015; 13 (2): 387-408. http://dx.doi.org/10.14204/ejrep.36.14075

29. Moreno B, Jiménez R, Gil A, Aspano MI, Torrero F. Análisis de la percepción del clima motivacional, necesidades psicológicas básicas, motivación autodeterminada y conductas de disciplina de estudiantes adolescentes en las clases de educación física. Eur. j. hum. mov. 2011; 26: 1-24. https://bit.ly/33lABG4

30. Babalis T. The relation of classroom climate to learning. J. Mod. Educ. Rev. 2013; 3(4): 289-301. http://bit.ly/31avTtr

31. Arguís R, Bolsas AP, Hernández S, Salvador MM. Programa Aulas Felices: la Psicología Positiva entra en las aulas. Revista del Museo Pedagógico de Aragón. 2011; (3): 52-7. http://bit.ly/2J4leub 